\title{
Three-dimensional impact angle guidance law based on robust repetitive control
}

\author{
Wenguang Zhang ${ }^{1}$ (D) $\cdot$ Wenjun $\mathrm{Yi}^{1} \cdot$ Jun Guan $^{1} \cdot \mathrm{Yue}_{\mathrm{Qu}}{ }^{1}$
}

Received: 27 June 2019 / Accepted: 31 October 2019 / Published online: 5 November 2019

(c) Springer Nature Switzerland AG 2019

\begin{abstract}
This paper presents a robust repetitive control (RC) design applied to three-dimensional homing guidance of missiles with impact angle constraint. The proposed guidance law is substantially a composite control method, which is constructed through a combination of RC and sliding mode control. More specifically, the RC exerts advantages to drive the state tracking error converge to zero, then sliding mode control is triggered, making the system be robust in terms of noise and disturbance. The effectiveness of the proposed guidance law is validated through simulation.
\end{abstract}

Keywords Guidance law · Impact angle $\cdot$ Repetitive control

\section{List of symbols}

$R$

$\phi$

$\theta$

$\vec{e}_{r}$

$\vec{e}_{\phi}$

$\vec{e}_{\theta}$

$\vec{a}_{T}=w_{r} \vec{e}_{r}+w_{\theta} \vec{e}_{\theta}+w_{\phi} \vec{e}_{\phi}$

$\vec{a}_{M}=u_{\theta} \vec{e}_{\theta}+u_{\phi} \vec{e}_{\phi}$

$\ddot{R}$

$\ddot{\phi}$

$\ddot{\theta}$

$\dot{R}$

$\dot{\theta}$

$\dot{\phi}$
Relative distance between the missile and the target Pitch line-of-sight angle (PLOS)

Yaw line-of-sight angle (YLOS)

Unit vector along the LOS

Unit vector along the PLOS

Unit vector along the YLOS

Acceleration vector of the target

Acceleration vector of the missile

Relative acceleration along to LOS

Angular acceleration along to LOS

Angular acceleration of $\theta$

Relative velocity between the missile and the target

Angular velocity of $\theta$

Angular velocity of $\phi$

\section{Introduction}

Intercepting maneuvering targets with a small miss-distance is not the only task of the guidance law design in some applications, for example, antitank or antiship missiles, which are also required to approach the target from a predetermined impact angle in order to increase the warhead effectiveness [1, 2]. Hence, it is necessary to design guidance law with impact angle constraint.

During the guidance process, the guidance system continuously measures the relative position information, and sends command to the flight control system. The kinematics equation of the missile-target pursuit dynamic behavior is found to be uncertain nonlinear multiple-input multiple-output (MIMO) system with cross-coupling [3]. In the past, Proportional Navigation Guidance law (PNG) was widely used in homing guidance area [4]. Along with the progress of computer science and mathematics, a lot of nonlinear control methods have been applied to this issue [5-8]. Among them, sliding mode control (SMC) was widely adopted by researchers for its unique properties, for example, it is robust to parameter variations and external disturbance

Wenguang Zhang, wgz8911@163.com | ${ }^{1}$ National Key Laboratory Transient Physics, Nanjing University of Science and Technology, Nanjing 210094, China. 
[9]. But SMC suffers from some drawbacks, which are: the upper bound of uncertainties must be known, and the existence of chattering phenomenon, which may cause the excitation of unmodeled dynamics [10].

Recently, a new guidance law design based on Iterative Learning Control (ILC) is proposed in [11], and the numerical experiments show that the proposed method is capable of reducing the time to reach the head-on condition to interception. However, impact angle constraint is not taken into consideration in this paper. Besides, there exists robustness problem of ILC [12].

In this paper, we propose a robust repetitive control strategy for guidance mission of homing missiles. The $\mathrm{RC}$ is combined with sliding mode control in order to acquire both of their advantages. Specifically, the RC is utilized to guarantee the reachability of the sliding mode, and then the sliding mode control is committed to enhance the robustness of the system. Simulations under different scenarios are performed, and the validation of the proposed method is verified.

This paper is organized as follows: in Sect. 2, the dynamics of target-missile relative motion is illustrated, and the object of the guidance law with impact angle constraint is addressed. In Sect. 3, the robust repetitive control is designed in the framework of sliding mode control. Numerical experiments are performed to demonstrate the effectiveness of the proposed method in Sect. 4. At last, concluding remarks are summarized in Sect. 5 .

\section{Problem formulation}

In the actual interception, the target-missile relative motion takes place in a three dimensional environment. It can be denoted in the spherical LOS coordinate system as Fig. 1 shows.

The 3D pursuit dynamic system can be expressed as follows [13]:

$\ddot{R}=R \dot{\phi}^{2}+R \dot{\theta}^{2} \cos ^{2} \phi+a_{T r}-a_{M r}$

$\ddot{\theta}=-\frac{2 \dot{R} \dot{\theta}}{R}+2 \dot{\phi} \dot{\theta} \tan \phi+\frac{a_{T \theta}}{R \cos \phi}-\frac{a_{M \theta}}{R \cos \phi}$

$\ddot{\phi}=-\frac{2 \dot{R} \dot{\phi}}{R}-\dot{\theta}^{2} \sin \phi \cos \phi+\frac{a_{T \phi}}{R}-\frac{a_{M \phi}}{R}$

In fact, only the accelerations normal to the missile's velocity are available in the terminal guidance phase. Therefore, only Eqs. (2) and (3) are used in guidance law design.

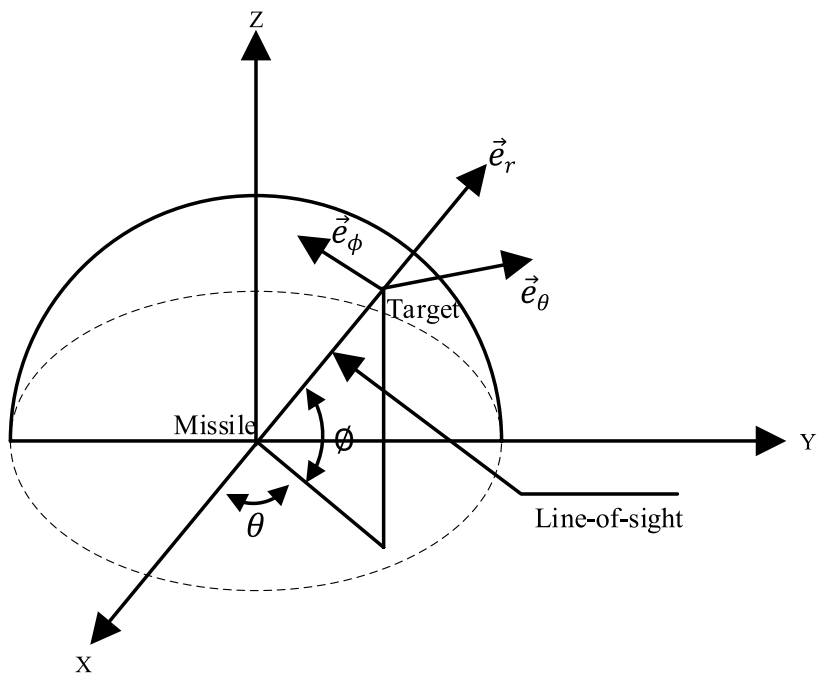

Fig. 1 Three-dimensional pursuit-evasion geometry

Assumption 1 [14] Assume that the missile intercepting the target by impact happens when $R=R_{0} \neq 0$, and there exist two positive constants $R_{\min }$ and $R_{\max }$, which satisfy $R_{\min }<R<R_{\max }$.

Let $\theta_{d}$ and $\phi_{d}$ be the desired final LOS angles in elevation and azimuth, respectively. By accepting the concept that zeroing the LOS angle rate will lead a perfect interception and taking the terminal angle constraint into consideration, the control object is to design a guidance law in such a way that $\theta \rightarrow \theta_{d}, \phi \rightarrow \phi_{d}, \dot{\theta} \rightarrow 0, \dot{\phi} \rightarrow 0$ can be fulfilled asymptotically [15].

\section{Composite guidance law design}

\subsection{Derivation of sliding surface}

Let $e_{\theta}$ and $e_{\phi}$ denote the tracking error of $\theta$ and $\phi$, respectively, which are defined as $e_{\theta}=\theta-\theta_{d}, e_{\phi}=\phi-\phi_{d}$. Then a sliding surface dynamics can be defined as follows:

$\sigma_{\theta}(t)=c_{1} e_{\theta}(t)+c_{2} \dot{e}_{\theta}(t)$

$\sigma_{\phi}(t)=c_{3} e_{\phi}(t)+c_{4} \dot{e}_{\phi}(t)$

where $c_{i},(i=1,2,3,4)$ are coefficients of a Hurwitz polynomial.

Computing the time derivative of Eqs. (4) and (5) and considering Eqs. (2) and (3) gives

$\frac{d}{d t}\left(\begin{array}{c}\sigma_{\theta} \\ \sigma_{\phi}\end{array}\right)=\left(\begin{array}{l}c_{1} \dot{\theta}+c_{2}\left(-\frac{2 \dot{R} \dot{\theta}}{R}+2 \dot{\phi} \dot{\theta} \tan \phi+\frac{a_{T \theta}}{R \cos \phi}-\frac{a_{M \theta}}{R \cos \phi}\right) \\ c_{3} \dot{\phi}+c_{4}\left(-\frac{2 \dot{R} \dot{\phi}}{R}-\dot{\theta}^{2} \sin \phi \cos \phi+\frac{a_{T \phi}}{R}-\frac{a_{M \phi}}{R}\right)\end{array}\right)$ 


\subsection{Robust RC guidance law design}

Let us consider the auxiliary control terms

$\left(\begin{array}{c}a_{M \theta} \\ a_{M \phi}\end{array}\right)=\left(\begin{array}{c}\frac{c_{1} \dot{\theta}-u_{1}}{c_{2}} R \cos \phi-2 \dot{R} \dot{\phi} \cos \phi+2 R \dot{\theta} \dot{\phi} \sin \phi \\ \frac{c_{3} \dot{\phi}-u_{2}}{c_{4}} R-2 \dot{R} \dot{\phi}-\frac{1}{2} R \dot{\theta}^{2} \sin 2 \phi\end{array}\right)$

where $u_{1}$ and $u_{2}$ are auxiliary controls. Then the system Eqs. (6) and (7) yields

$\frac{d}{d t}\left(\begin{array}{c}\sigma_{\theta} \\ \sigma_{\phi}\end{array}\right)=\left(\begin{array}{l}u_{1}+w_{\theta} \\ u_{2}+w_{\phi}\end{array}\right)$

where $w_{\theta}=c_{2} \frac{a_{T \theta}}{R \cos \phi}$ and $w_{\phi}=c_{4} \frac{a_{T \phi}}{R}$ belong to be uncertain terms for reason that they contain acceleration information of the target, which cannot be measured. According to the research in [11], we can assume that

$w_{\theta}=\eta_{\theta}(t) \sigma_{\theta}$

$w_{\phi}=\eta_{\phi}(t) \sigma_{\phi}$

where $\eta_{\theta}(t), \eta_{\phi}(t)$ are unknown variables. By substituting Eqs. (9) and (10) into system (8), we can obtain

$\frac{d}{d t}\left(\begin{array}{c}\sigma_{\theta} \\ \sigma_{\phi}\end{array}\right)=\left(\begin{array}{c}u_{1}+\eta_{\theta}(t) \sigma_{\theta} \\ u_{2}+\eta_{\phi}(t) \sigma_{\phi}\end{array}\right)$

The reference trajectory for system (11) is generated by the following dynamics:

$y_{1}(t)=0$

$y_{2}(t)=0$

Enlightened by the works in $[11,16]$, we design the robust $\mathrm{RC}$ control law as follows:

$u_{1}=k_{1}\left(y_{1}-\sigma_{\theta}\right)-\hat{\eta}_{\theta}(t) \sigma_{\theta}$

$u_{2}=k_{2}\left(y_{2}-\sigma_{\phi}\right)-\hat{\eta}_{\phi}(t) \sigma_{\phi}$

$\hat{\eta}_{\theta}(t)=\operatorname{proj}\left(\hat{\eta}_{\theta}\left(t-T_{\eta}\right)\right)-\sigma_{\theta}\left(y_{1}-\sigma_{\theta}\right)$

$\hat{\eta}_{\phi}(t)=\operatorname{proj}\left(\hat{\eta}_{\phi}\left(t-T_{\eta}\right)\right)-\sigma_{\phi}\left(y_{2}-\sigma_{\phi}\right)$

$\operatorname{proj}\left(\hat{\eta}_{\theta}\left(t-T_{\eta}\right)\right)=\left\{\begin{array}{c}\hat{\eta}_{\theta}\left(t-T_{\eta}\right),\left|\hat{\eta}_{\theta}\left(t-T_{\eta}\right)\right| \leq \eta_{\theta}^{*} \\ \operatorname{sign}\left[\hat{\eta}_{\theta}\left(t-T_{\eta}\right)\right] \eta_{\theta^{\prime}}^{*}\left|\hat{\eta}_{\theta}\left(t-T_{\eta}\right)\right|>\eta_{\theta}^{*}\end{array}\right.$

$\operatorname{proj}\left(\hat{\eta}_{\phi}\left(t-T_{\eta}\right)\right)=\left\{\begin{array}{c}\hat{\eta}_{\phi}\left(t-T_{\eta}\right),\left|\hat{\eta}_{\phi}\left(t-T_{\eta}\right)\right| \leq \eta_{\phi}^{*} \\ \operatorname{sign}\left[\hat{\eta}_{\phi}\left(t-T_{\eta}\right)\right] \eta_{\phi^{\prime}}^{*}\left|\hat{\eta}_{\phi}\left(t-T_{\eta}\right)\right|>\eta_{\phi}^{*}\end{array}\right.$

where $\eta_{\theta}^{*}$ and $\eta_{\phi}^{*}$ are design parameters, $T_{\eta}$ is value of period for updating $\hat{\eta}_{\theta}$ and $\hat{\eta}_{\phi}$.

\subsection{Stability analysis}

In this section, we will prove the system represented by Eq. (11) is stable by Lyapunov stable theory [16]. Because the dynamic models of $\sigma_{\theta}$ and $\sigma_{\phi}$ have the similar forms, here we only take $\sigma_{\theta}$ as an example to show the process of proof.

Firstly, the tracking error of $\sigma_{\theta}$ is defined as $e_{1}(t)=y_{1}-\sigma_{\theta}$, and the initial condition of $e_{\theta}$ can be characterised by the following assumption:

Assumption $2 e_{1}(0)$ is random and bounded by a constant $C$.

Differentiating $e_{\theta}$ with time and substituting the control law Eq. (14) we obtain

$\dot{e}_{1}=-k_{1} e_{1}-\phi(t) \sigma_{\theta}$

where $\phi(t) \triangleq \eta_{\theta}(t)-\hat{\eta}_{\theta}(t)$.

Define the following Lyapunov functional:

$V\left(\sigma_{\theta}, \phi(t), \phi(t-T), t\right)=\frac{1}{2} e_{1}^{2}+\frac{1}{2} \int_{0}^{t} \phi^{2}(\tau) d \tau+\frac{1}{2} \int_{t}^{T} \phi^{2}(\tau-T) d \tau$

where $T$ is repetitive control cycle.

The derivative with time $t$ of $V\left(\sigma_{\theta}, \phi(t), \phi(t-T), t\right)$ is

$\dot{V}\left(\sigma_{\theta}, \phi(t), \phi(t-T), t\right)=e_{1} \dot{e}_{1}+\frac{1}{2}\left(\phi^{2}(t)-\phi^{2}(t-T)\right)$

Substituted the error dynamics Eq. (20), the first term on the right-hand side of Eq. (22) can be described by

$e_{1} \dot{e}_{1}=-\phi(t) \sigma_{\theta} e_{1}-k_{1} e_{1}^{2}$

Next, substituting Eq. (16) into the other term on the right-hand side of Eq. (22), utilizing the relations $(a-b)^{2}-(a-c)^{2}=-2(a-b)(b-c)-(b-c)^{2}$ and the $\operatorname{property}\left(\eta_{\theta}(t)-\hat{\eta}_{\theta}(t)\right)^{2} \geq\left(\eta_{\theta}(t)-\operatorname{proj}\left(\hat{\eta}_{\theta}\left(t-T_{\eta}\right)\right)\right)^{2}$ for any $\hat{\eta}_{\theta}(t)$, we get

$$
\begin{aligned}
\frac{1}{2}( & \left.\phi^{2}(t)-\phi^{2}(t-T)\right) \\
= & \frac{1}{2}\left[\left(\eta_{\theta}-\hat{\eta}_{\theta}(t)\right)^{2}-\left(\eta_{\theta}-\hat{\eta}_{\theta}\left(t-T_{\eta}\right)\right)^{2}\right] \\
\leq & \frac{1}{2}\left[\left(\eta_{\theta}-\hat{\eta}_{\theta}(t)\right)^{2}-\left(\eta_{\theta}-\operatorname{proj}\left(\hat{\eta}_{\theta}\left(t-T_{\eta}\right)\right)\right)^{2}\right] \\
= & -\left(\eta_{\theta}-\hat{\eta}_{\theta}\right)\left(\hat{\eta}_{\theta}-\operatorname{proj}\left(\hat{\eta}_{\theta}\left(t-T_{\eta}\right)\right)\right) \\
& -\frac{1}{2}\left(\hat{\eta}_{\theta}-\operatorname{proj}\left(\hat{\eta}_{\theta}\left(t-T_{\eta}\right)\right)\right)^{2} \\
= & \phi(t) \sigma_{\theta} e_{1}-\frac{1}{2} \sigma_{\theta}^{2} e_{1}^{2}
\end{aligned}
$$

Therefore, the derivative of $V\left(\sigma_{\theta}, \phi(t), \phi(t-T), t\right)$ is 
$\dot{V}\left(\sigma_{\theta}, \phi(t), \phi(t-T), t\right)=-k_{1} e_{1}^{2}-\frac{1}{2} \sigma_{\theta}^{2} e_{1}^{2}<0$

According to Lyapunov stable theory and taking Assumption 2 into consideration, $e_{1}$ is convergent, which means $\left|e_{1}(t+T)\right|$ is less than $\left|e_{1}(t)\right|$. If $t$ is large enough, $e_{1}$ will be able to approach to origin, which means $y_{1}-\sigma_{\theta}=0$ will be guaranteed. Using Eq. (12), $\sigma_{\theta}$ is able to converge to zero. In other words, the sliding mode is reachable. As previously mentioned, the constants $c_{i}$ are Hurwitz polynomial, hence $e_{\theta}$ and $e_{\phi}$ as well as their derivatives converge to zero asymptotically during the sliding mode, and the system is able to keep invariant to noise and disturbance. Therefore, the system (11) is stable.

Remark 1 Note that the guidance law proposed in this paper is discontinuous because of the presence of signum functions, leading to chattering phenomenon. To weaken this phenomenon, $\operatorname{proj}\left(\hat{\eta}_{\theta}\left(t-T_{\eta}\right)\right)$ and $\operatorname{proj}\left(\hat{\eta}_{\phi}\left(t-T_{\eta}\right)\right)$ can be replaced by

$k_{3}\left|\operatorname{proj}\left(\hat{\eta}_{\theta}\left(t-T_{\eta}\right)\right)\right|^{\frac{2}{3}} \operatorname{sign}\left(\operatorname{proj}\left(\hat{\eta}_{\theta}\left(t-T_{\eta}\right)\right)\right)$

$k_{4}\left|\operatorname{proj}\left(\hat{\eta}_{\phi}\left(t-T_{\eta}\right)\right)\right|^{\frac{2}{3}} \operatorname{sign}\left(\operatorname{proj}\left(\hat{\eta}_{\phi}\left(t-T_{\eta}\right)\right)\right)$

respectively.

\section{Simulation results}

\subsection{Numerical experiments under different scenarios}

To verify the effectiveness of the proposed robust repetitive control based guidance law (RRCGL), the following two cases for the different target accelerations are considered as follows:

\section{Case 1}

$a_{T r}=50 \sin (0.5 \pi t)$

$a_{T \theta}=-50 \sin (0.5 \pi t)$

$a_{T \phi}=-60 \sin (2 \pi t+\pi / 3)$

\section{Case 2}

$a_{T r}=-30$

$a_{T \theta}=30$

$a_{T \phi}=30$
Table 1 Initial conditions for the two cases

\begin{tabular}{ll}
\hline$r(0)$ & $12,000 \mathrm{~m}$ \\
$\theta(0)$ & $\pi / 4 \mathrm{rad}$ \\
$\phi(0)$ & $\pi / 4 \mathrm{rad}$ \\
$V_{r}(0)$ & -900 \\
$V_{\theta}(0)$ & 400 \\
$V_{\phi}(0)$ & 500 \\
\hline
\end{tabular}

The parameters of the controller are set to be: $k_{1}=0.6$, $k_{2}=0.1, k_{3}=10, k_{4}=10, \gamma_{1}=10, \gamma_{2}=10, \theta_{d}=70 \mathrm{deg}$, $\phi_{d}=60 \mathrm{deg}, c_{1}=0.95, c_{2}=1.6, c_{3}=0.32, c_{4}=0.6$, $T_{\eta}=0.5 \mathrm{~s}, T=0.01 \mathrm{~s}$, and the initial conditions are listed in Table 1.

Where $V_{r}(t), V_{\theta}(t)$ and $V_{\phi}(t)$ in Table 1 are defined as

$V_{r}=\dot{r}$

$V_{\theta}=r \dot{\theta} \cos \phi$

$V_{\phi}=r \dot{\phi}$

For performance comparison, the ILCGL in Ref. [11] and NTSMGL in Ref. [15] are simulated under the same conditions.

\subsubsection{Simulation results for case 1}

For case 1, Fig. 2 shows the simulation results. As demonstrated in Fig. 2a, all the three methods enable successful interceptions, and it should be noted that the two methods named RRCGL and ILCGL [11] cost less interception time than NTSMGL [15] method. From Fig. 2b, $c$, we can observe that the sliding mode variables of the proposed guidance law converge to zero faster than those of NTSMGL does. Besides, the large initial error for $s$ denotes that NTSMGL method demands large control gains to guarantee an effective interception. As presented in Fig. 2d, e, both of RRCGL and NTSMGL have the ability to impose the demanded impact angle, but ILCGL cannot drive the angles to the demanded values. From Fig. 2f, g, we can note that the missile acceleration produced by NTSMGL experiences a severe chattering phenomenon. On the contrary, RRC generates much more smoother control signal. As Fig. 2h, i show, parameters updated by Eqs. (16-19) change greatly around $2 \mathrm{~s}$, which is the reason why the control signal has a sudden change around that moment. It should be noted that the sliding mode variables just enter the sliding mode at that moment. After that, the parameters change slightly between each iteration intervals. 


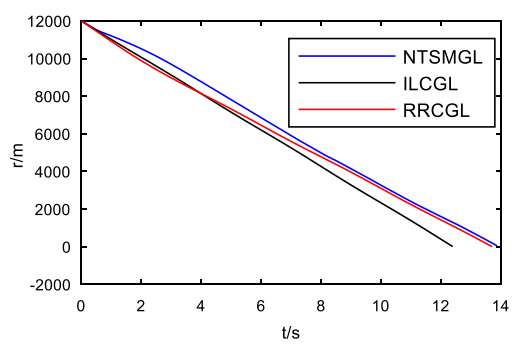

(a) Relative distance between the missile and the target

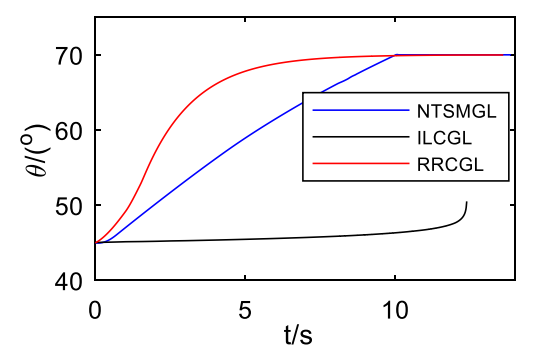

(d) Yaw line-of-sight angle

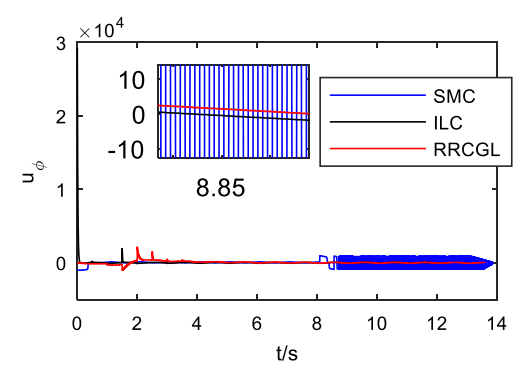

(g) Missile acceleration in elevation loop

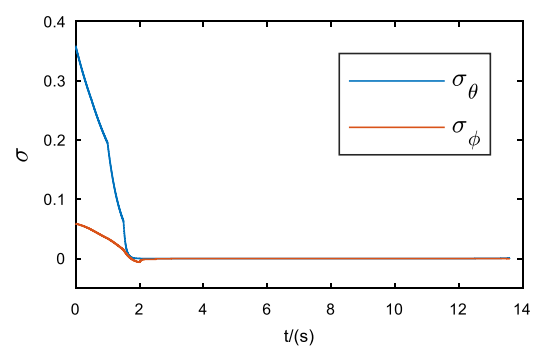

(b) Sliding mode manifold for $\sigma$

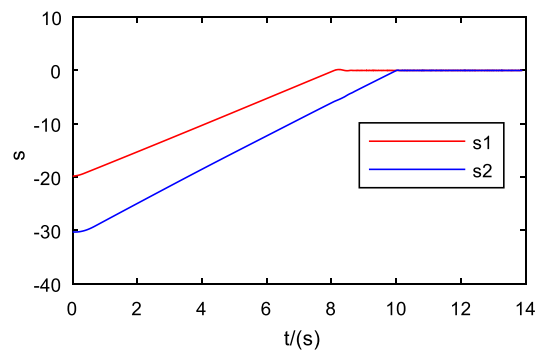

(c) Sliding mode manifold for $\mathrm{s}$ in

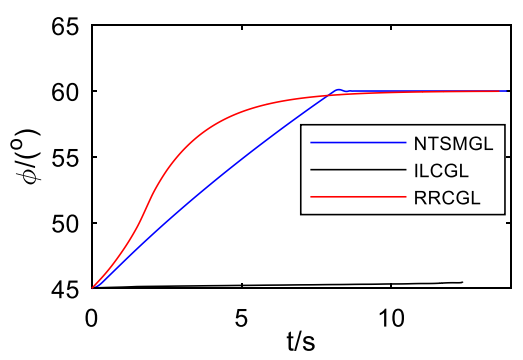

(e) Pitch line-of-sight angle

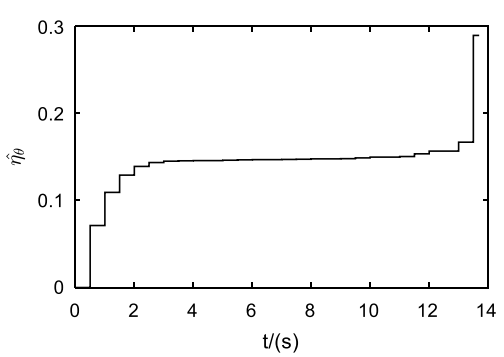

(h) parametric updating evolution for $\hat{\eta}_{\theta}$

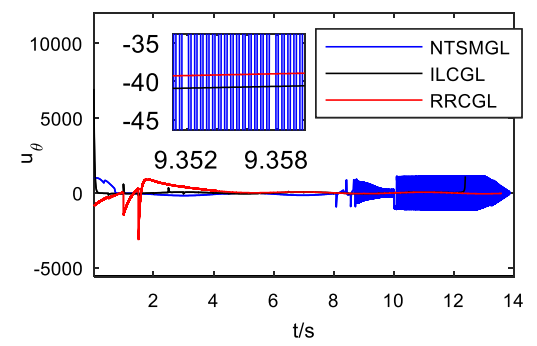

(f) Missile acceleration in azimuth loop

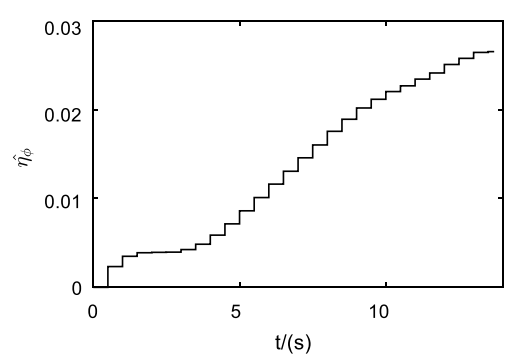

(i) Parametric updating evolution for $\hat{\eta}_{\phi}$

Fig. 2 Simulation results for case 1

\subsubsection{Simulation results for case 2}

To cope with the interceptor maneuvering in this way, RRCGL and ILCGL still spend less time than NTSMGL to complete the intercept mission as Fig. 3a shows. Figure 3b, c depict the sliding manifold for RRCGL and NTSMGL, respectively. Obviously, RRCGL drives the sliding variables to zero much more quickly than NTSMGL does. The manifold of s2 even fluctuates during the process of convergence. As a result to this, the proposed method makes the angles converge to the demanded values faster than NTSMGL does, just as Fig. 3d, e show. Meanwhile, ILCGL method still has no ability to impose the demanded impact angles. As presented in Fig. 3f, g, NTSMGL still suffers from chattering problem in this scenario. To the contrary, the acceleration profiles generated by the other two methods are smooth on the whole. Figures $3 \mathrm{~h}$, i show the parametric updating evolution for $\hat{\eta}_{\theta}$ and $\hat{\eta}_{\phi}$. It can be concluded that the proposed guidance law is more robust against the target maneuver than the other two methods.

\section{Conclusion}

In this paper, a novel composite guidance law based on RC and sliding mode control is proposed. The RC is integrated to guarantee the reachability of the sliding mode, during which the variables of interest converge 


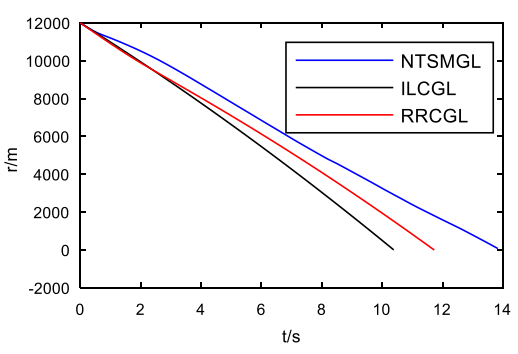

(a) Relative distance between the missile

(b) and the target

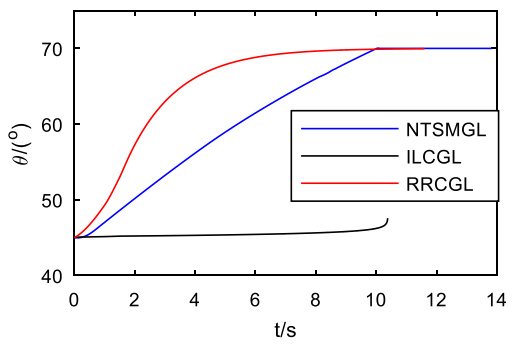

(d) Yaw line-of-sight angle

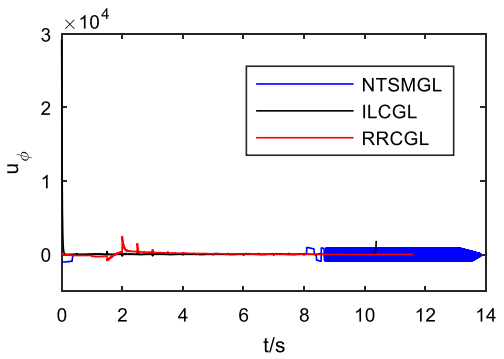

(g) Missile acceleration in elevation loop $\hat{\eta}_{\phi}$

Fig. 3 Simulation results for case 2

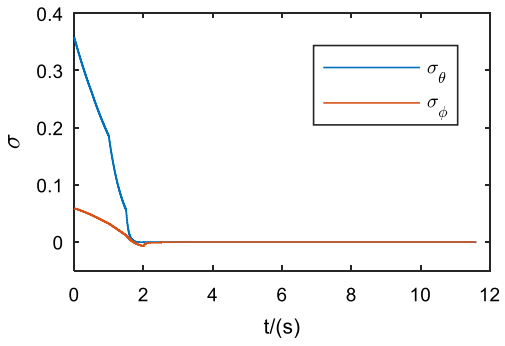

(b) Sliding mode manifold for $\sigma$

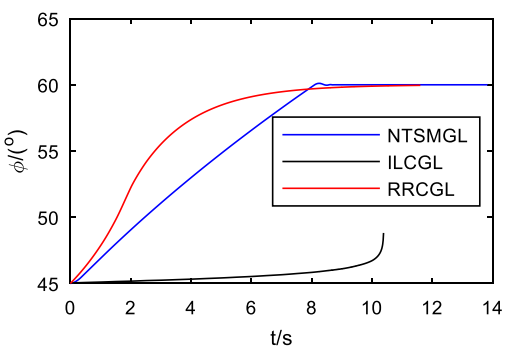

(e) Pitch line-of-sight angle

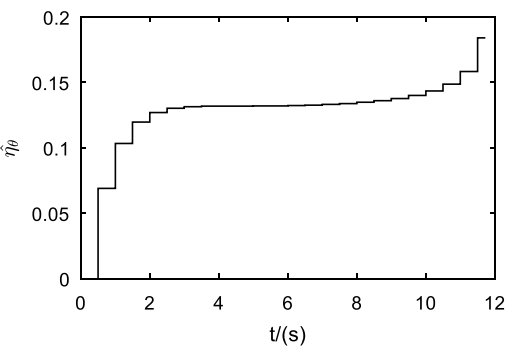

(h) parametric updating evolution for $\hat{\eta}_{\theta}$

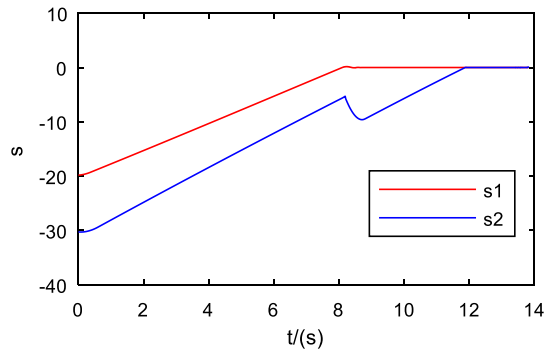

(c) Sliding mode manifold for $\mathrm{s}$ in Ref. [15].

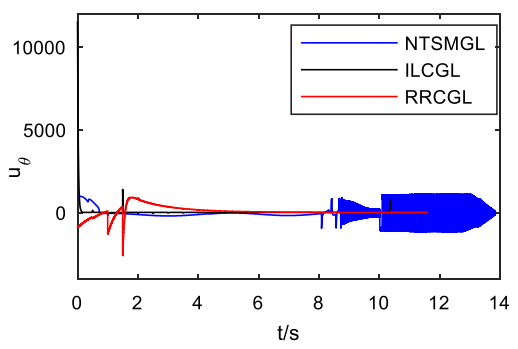

(f) Missile acceleration in azimuth loop

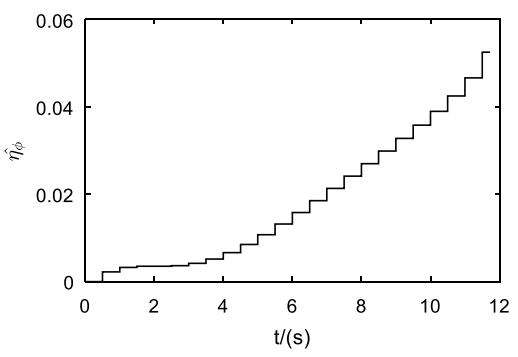

(i) Parametric updating evolution for to the demanded values asymptotically. Numerical simulations show that the proposed guidance law is robust to extreme forms of target maneuver, not only the target can be intercepted, but also the demanded impact angles are satisfied.

Funding This work was supported in part of China Postdoctoral Science Foundation under Grant of 2019M651838.

\section{Compliance with ethical standards}

Conflict of interest The authors declare that they have no conflict of interest.

\section{References}

1. Erer KS, Merttopçuoglu O (2012) Indirect impact angle control against stationary targets using biased pure proportional navigation. J Guid Control Dyn 35:700-704

2. Hou Z, Liu L, Wang $Y$ et al (2016) Terminal impact angle constraint guidance with dual sliding surfaces and model free target acceleration estimator. IEEE Trans Control Syst Technol 25:85-100

3. Song J, Song S (2016) Three-dimensional guidance law based on adaptive integral sliding mode control. Chin J Aeronaut 29:202-214

4. Zarchan $P$ (1994) Tactical and strategic missile guidance. AIAA, Reston, VA, 1997, pp 161-180 
5. Shi L, Zhu ZH, Tang S, Yan X (2017) Prescribed performance slide mode guidance law with terminal line-of-sight angle constraint against maneuvering targets. Nonlinear Dyn 88:2101-2110

6. Peng Z, Wang J, Dan W (2017) Distributed maneuvering of autonomous surface vehicles based on neurodynamic optimization and fuzzy approximation. IEEE Trans Control Syst Technol 99:1-8

7. Wang X, Tan CP, Zhou D (2018) Observer-based PIGC for missiles with impact angle constraint. IEEE Trans Aerosp Electron Syst 55(5):2226-2240

8. Zhao Y, Sheng Y, Liu X (2016) Impact angle constrained guidance for all-aspect interception with function-based finite-time sliding mode control. Nonlinear Dyn 85:1791-1804

9. Utkin VI (2002) Sliding mode control design principles and applications to electric drives. IEEE Trans Ind Electron 40:23-36

10. Dhadekar DD, Patre BM (2016) UDE-based decoupled full-order sliding mode control for a class of uncertain nonlinear MIMO systems. Nonlinear Dyn 88:263-276

11. Acho $L$ (2017) Iterative learning control for homing guidance design of missiles. Def Technol 13:40-46
12. Norrlöf Mikael, Gunnarsson S (2002) Time and frequency domain convergence properties in iterative learning control. Int J Control 75:1114-1126

13. Yang CD, Yang CC (1996) Analytical solution of three-dimensional realistic true proportional navigation. J Guid Control Dyn 19:569-577

14. Shtessel YB, Shkolnikov IA, Levant A (2009) Guidance and control of missile interceptor using second-order sliding modes. IEEE Trans Aerosp Electron Syst 45:110-124

15. Kumar SR, Ghose D (2017) Three-dimensional impact angle guidance with coupled engagement dynamics. Proc IMechE Part G: J Aerosp Eng 231(4):621-641

16. Xu JX, Yan R (2005) On initial conditions in iterative learning control. IEEE Trans Autom Control 50:1349-1354

Publisher's Note Springer Nature remains neutral with regard to jurisdictional claims in published maps and institutional affiliations. 